Dear Author,

Please, note that changes made to the HTML content will be added to the article before publication, but are not reflected in this PDF.

Note also that this file should not be used for submitting corrections. 


\title{
Q1 Preventing miscarriages of justice: A review of forensic firearm identification
}

\author{
Q2 Rachel S. Bolton-King *
}

Science Centre, Department of Forensic and Crime Science, Staffordshire University, Leek Road, Stoke-on-Trent, Staffordshire, ST4 2DF England, UK

\section{A R T I C L E I N F O}

\section{Article history:}

Received 12 October 2015

Accepted 8 November 2015

Available online $\mathrm{xxxx}$

\section{Keywords:}

Competence

Evidence

Firearm identification

Forensic

Imaging

Miscarriage of justice

\begin{abstract}
A B S T R A C T
The role of a firearm examiner is wide ranging, involving tasks that require scientific understanding in aspects of 18 chemistry, physics and biology. This article aims to provide a critical review of the key scientific principles and 19 practices specifically involved with forensic firearm identification and to discuss how misidentifications have re- 20 sulted in cases of injustice. Implementation of quality assured examination practice, demonstration of individual 21 examiner competence and more objective methods of reporting are being adopted by firearm examiners and lab- 22 oratories to address some of the criticisms relating to subjectivity and standardisation inherent within the disci- 23 pline. The impact of these changes is outlined and further recommendations are made for both examiners and 24 legal professionals to minimise the potential for future injustices involving firearms evidence. Latest research 25 in the field is cited, continuing to support the theory and use of firearm identification as admissible evidence 26 in court.
\end{abstract}

(c) 2015 Published by Elsevier Ireland Ltd. on behalf of The Chartered Society of Forensic Sciences.

\section{Introduction}

Between 2008 and 2009, a number of reports were published [1-3] regarding the position of forensic science, current issues and the recommendations that need to be made in specific forensic disciplines going forward. Of these, the National Academy of Science (NAS) report [2] has been the most widely cited reference, especially referred to by legal professionals and the media, to criticise and undermine work undertaken by forensic practitioners when applying their scientific interpretations and discipline knowledge to casework in the pursuit of justice. In particular, this report identified concerns regarding the scientific underpinning of pattern recognition based disciplines such as fingerprints, firearms and questioned documents. These fields were highlighted due to the perception that there were limited published research and documentation to support the validity and reliability of the science and the interpretations made following forensic analysis.

In response to the NAS [2] report, the American Society of Crime Laboratory Directors (ASCLD [4]) identified the two fundamental issues highlighted by NAS; 1 ) the lack of standardisation of procedure across laboratories within disciplines and 2) the need for more resources, education and training for practitioners to carry out casework. Both of these issues are typically caused due to the lack of stable and sufficient funding in the United States (US). However, these issues apply in other countries worldwide and are significant causes of miscarriages of justice (Section 2), negatively impacting confidence in forensic

\footnotetext{
* Tel.: +441782294367.

E-mail address: r.bolton-king@staffs.ac.uk.
}

evidence presented in court. The ASCLD [4] response therefore high- 57 lights the need for experts in the forensic community to be fully pre- 58 pared to answer questions in the courtroom and provide evidence to 59 document, justify and support the scientific underpinning and validity 60 of the analytical methods utilised within their disciplines.

Other organisations, such as California Association of Criminalists 62 (CAC [5]) also responded to the NAS report, with only non-expert re- 63 ports such as the National Association of Criminal Defense Lawyers 64 (NACDL [6]) predominantly agreeing with the NAS [2] report outcomes. 65 The ASCLD response [4] highlights that to change these perceptions the 66 discipline experts need to engage and collaborate with non-experts, 67 such as legal professionals, to communicate the science underpinning 68 our fields. Although, to achieve a successful outcome this engagement 69 needs to be a two way process. Professionals using information provid- 70 ed by subject experts need to ask the right questions to ensure their 71 understanding of the capabilities and limitations of the science and in- 72 terpretation supporting casework is presented in an unbiased way to 73 those in the courtroom, especially jurors.

One of the most basic and fundamental issues experienced by this 75 field is the common misuse of the term 'forensic ballistics' to holistically 76 cover the three core disciplines; forensic firearm examination, firearm 77 identification and ballistics. These three areas are quite separate scien- 78 tific concepts for which different articles could be written. Forensic fire- 79 arm examination covers the examination of firearms to evaluate their 80 forensic value and establish their functionality. Forensic firearm identi- 81 fication involves the comparison of fired ammunition components to 82 test fired exemplars from a suspected firearm. Ballistics relates to the 83 motion of the projectile from the time the ammunition is fired until 84 
Firing angle determination shooting distances.

Table 1 the moment it comes to rest. Ballistics is further sub-divided into three key areas; internal (everything that happens to the projectile before it leaves the weapon), external (the motion of the projectile as it is travelling through the atmosphere) and terminal ballistics (the motion of the projectile once it comes into contact with matter of any kind). Intermediate ballistics is sometimes considered as an additional component of ballistics that covers the motion of the projectile just as it exits the muzzle of the barrel until it escapes the flow of gases and enters free flight. The identification of gunshot residue (GSR) as coming from firearm ammunition using analytical chemistry techniques such as scanning electron microscopy and energy dispersive x-ray (SEM EDX) is an additional area of expertise sometimes considered within 'forensic ballistics'. Firearm examiners are typically not qualified to identify primer particles as being GSR, however, they may be asked to interpret GSR evidence from unburnt and partially burnt gunpowder to estimate

The field of firearms and ballistics is extensive and truly multidisciplinary, requiring the firearm examiner to be knowledgeable and demonstrate depth of understanding across all three core sciences (chemistry, physics and biology) and apply mathematics to compare, analyse, interpret and link shooting related incidents. Typical tasks that a firearm examiner may be asked to carry out and be called upon as an expert witness are summarised in Table 1 . However, it is important to appreciate that a single firearm examiner may not have the training and experience to carry out all of these roles due to the broad nature of the discipline. Tasks associated with firearm examination and function testing, legal classification and firearm identification may be considered the more common skills. Shooting incident reconstruction and serial number restoration may be considered as specialised areas of the field.

There are numerous recommended textbooks [7-11] designed for reading by both laypeople and experienced examiners, which discuss each of these scientific areas in significantly greater depth. The purpose of this article is therefore not to summarise those already written, but to provide a critical reflection on the causes of miscarriages in justice (Section 2) in the area of forensic firearm identification (Section 3) and discuss how professionals are trying to minimise the occurrence of these occurring in the future (Section 4).

\section{Miscarriages of justice}

Miscarriages of justice are undoubtedly damaging to the lives of those wrongfully convicted, the victim of the crime as well as the families, friends and associates of these parties. In comparison to the number of 125 those correctly and successfully convicted for the crimes they have com- 126 mitted, known injustices are typically infrequent. The criminal justice 127 system and criminal proceedings involve a number of key parties who 128 all have a role to play in ensuring that the outcome is appropriate to 129 the gathered evidence and intelligence presented in the courtroom. 130 Expert witness evidence is only one element of this wide network of in- 131 dividuals. The judge, lawyers (both prosecution and defence) and impor- 132 tantly the members of the jury (if applicable) are all pivotal and 133 responsible for reducing the probability of a miscarriage of justice occur- 134 ring, however, this chance will never be eliminated. The judge should act 135 as gatekeeper to ensure only appropriate expert witnesses present ad- 136 missible evidence and legal counsel need to ensure they do not make as- 137 sumptions about the work undertaken by the expert. When undertaking 138 cross-examinations counsel should fully question the procedures and 139 outcomes presented and should examine the expert's training, experi- 140 ence and competence prior to and during proceedings. 141

Over recent years there has been more emphasis and importance 142 realised in the legal community about the requirement for them to be- 143 come educated and understand the science underpinning forensic evi- 144 dence. The jury however, do not have this luxury and therefore it is 145 critical that those involved with cross-examination and presentation 146 of expert testimony in court provide opportunities for experts to com- 147 municate the necessary science effectively to laypersons. Such commu- 148 nication needs to be to an extent whereby each juror sufficiently 149 understands the evidence, how it has been interpreted and the signifi- 150 cance of the interpretation in the context of the case. Jurors get limited 151 opportunities to clarify their understanding of scientific evidence and 152 therefore it is vital that legal counsel ask expert witnesses the right 153 questions to ensure that jurors correctly interpret and weight the infor- 154 mation presented to them by both the prosecution and defence. 155

Due to the limitations of firearms evidence (Section 3), a specific 156 person under suspicion for a crime cannot be attributed by analysis of 157 firearms evidence alone. This means that information provided by fire- 158 arms evidence is typically corroborative rather than conclusive; there 159 must be other forms of physical or intelligence-based evidence to in- 160 crease the probability that one particular individual was more likely to 161 have committed the crime than another. Each case of miscarriage of jus- 162 tice may therefore involve a number of contributing factors that result- 163 ed in an incorrect verdict, not just related to the interpretation of 164 firearms evidence. For example, in a case where a suspect weapon is re- 165 covered and can be test-fired for comparison against recovered crime 166

Tasks potentially requested to be undertaken by a firearm examiner.

\begin{tabular}{|c|c|}
\hline Task & Purpose(s) of task \\
\hline Firearms classification & $\begin{array}{l}\text { Identify the legal classification of a firearm (or component) within the local region's firearms legislation to determine } \\
\text { whether any charges should be brought by law enforcement for the possession of a firearm }\end{array}$ \\
\hline Function testing & $\begin{array}{l}\text { Determine whether the firearm is functioning as per manufacturer's design, whether it has been modified or converted, } \\
\text { deactivated or reactivated and/or the capability of accidental discharge }\end{array}$ \\
\hline Firearm restoration & Restore a firearm to its functional order so that the weapon can be test-fired \\
\hline Serial number restoration & $\begin{array}{l}\text { Recover obliterated serial number(s) on the firearm components, which can determine the date of manufacture, } \\
\text { and trace the current and previous owner of the firearm }\end{array}$ \\
\hline Test-firing & $\begin{array}{l}\text { Create fired ammunition components (e.g. bullets and/or cartridge cases) from a known firearm for reference and } \\
\text { forensic comparison purposes }\end{array}$ \\
\hline Forensic firearms identification & $\begin{array}{l}\text { 1. Identify whether fired ammunition components recovered from a crime scene have been fired from a specific } \\
\text { suspect weapon by comparing to known reference samples test-fired from the weapon } \\
\text { 2. Determine the number of firearms used in a shooting incident } \\
\text { 3. Link crime scenes together by comparing fired ammunition components (evidence samples) from multiple scenes }\end{array}$ \\
\hline Firing angle determination & $\begin{array}{l}\text { 1. Identify the angle of the projectile impact (e.g. using bullet hole or ricochet mark) } \\
\text { 2. Identify the direction of the projectile impact } \\
\text { 3. Determine the possible firing location }\end{array}$ \\
\hline Muzzle-to-target distance determination & $\begin{array}{l}\text { Determine how far away (range) the muzzle of the gun was positioned from the target/victim by comparing known } \\
\text { results from simulated crime scenes }\end{array}$ \\
\hline Trajectory analysis & $\begin{array}{l}\text { 1. Estimate the range of fire and thus determine where the projectile (e.g. bullet) may be located } \\
\text { 2. Determine the firing location } \\
\text { 3. Establish whether the firing location is consistent with the terminal location of the projectile } \\
\text { 4. Confirm or refute eyewitness testimony }\end{array}$ \\
\hline
\end{tabular}


scene evidence, forensic firearm identification and shooting incident reconstruction can only identify the tool (i.e. specific firearm) and manner in which the shooting incident occurred respectively. The interpretation of this evidence cannot solely be used to determine when the incident occurred and/or identify the individual who did the shooting-this would require additional expertise in DNA (deoxyribonucleic acid) or fingerprint evidence together with other corroborative evidence and intelligence from other sources e.g. CCTV (closed circuit television), eyewitness testimony etc.

With respect to criminal cases involving firearms evidence there have been a number of successful appeals resulting in quashed convictions. There have also been instances where convictions have been quashed due to the recovery of new firearms evidence or request for further testing of evidence not originally requested by the investigation. These cases have typically been associated with four aspects of firearms evidence; legal classification of a weapon as a firearm, forensic firearm identification, identification of gunshot residue and bullet lead analysis. The scope of this article only considers firearm identification.

Understanding the root cause of miscarriages of justice involving multiple types of evidence are not well discussed in literature and can be complex to determine, even after reading transcript summaries. In the media, injustice is frequently reported as unreliable or flawed ballistics evidence yet they typically provide no explanation for why the evidence was deemed unreliable. Such claims may also be misreported as the major cause when there may have been other major factors involved when reaching an incorrect verdict. Cates [12] summarises the 1989 case of Troy Davies vs State of Georgia highlighting 'unreliable ballistics evidence' as the cause of the injustice and also mentioning errors in eyewitness testimony. However, it is possible that during the decision making process the original verdict was reached by more heavily weighting eyewitness testimony rather than firearms evidence.

Instances where miscarriages of justice have primarily resulted from incorrect forensic firearm identification are illustrated by casework undertaken in a number of US police crime laboratories including Detroit $[13,14]$, Boston [15] and Houston [16]. Initial review of the Boston crime laboratory identified that $10 \%$ of 200 sampled cases resulted in a misidentification of a specific weapon to the recovered scene evidence. Although, due to the corroborative nature of firearms evidence one should not assume that those cases of misidentification actually resulted in any miscarriages of justice; there is of course the potential for there to have been some. Without having direct access to the evidence and trial transcripts in each of these cases, the author cannot go into further detail about specifically how these misidentifications impacted in each case outcome. Investigations within each of the three crime laboratories typically found issues predominantly arising from insufficient training and experience of the individuals conducting forensic firearm identification. Without appropriate depth of understanding regarding the scientific theory that underpin firearm identification and the impact of a wide range of variables influencing the ability to identify firearms, there is a much higher probability of an individual making an incorrect conclusion regarding firearms evidence. Training and experience of forensic firearm examiners is therefore of critical importance to accurately interpret and compare firearms evidence and these aspects will be further discussed in Section 3.

Over recent years there have been criticisms made regarding the variability in methods used in casework and potentially overemphasised strength of the conclusions made by examiners 'to the exclusion of all others', potentially resulting in miscarriages of justice. However, improvements in standardisation of analysis procedures, reporting and training in expert witness testimony have started to address these concerns and improvements are continuing to be implemented (Section 4.4). It is important that examiners follow the laboratory's standard operating processes in routine casework. However, there are times when general protocols are insufficient or inappropriate and additional testing or variations in protocol are required. In such cases it is imperative that the method employed is fully documented and justified so that the records can be subject to peer- 233 review during criminal proceedings. Lack of disclosure of evidential 234 findings and/or incomplete recording of examination methods and in- 235 terpretations can understandably raise questions over reliability and ac- 236 curacy of evidence presented leading to appeals and writs of habeas 237 corpus, as demonstrated by Bernal vs Dretke [17,18].

Any instance of miscarriage of justice will taint the reputation of the 239 use of relevant scientific evidence and put into question the reliability of 240 the opinions presented by the majority of very experienced and fully 241 trained individuals in that field. Humans are and should be at the 242 heart of all final conclusions regarding forensic analysis and interpreta- 243 tion of evidence. Even when technology and consistent procedures are 244 used to support their conclusions, human input will have been neces- 245 sary to setup equipment, carry out the procedure and make judgements 246 based on prior experience and knowledge, therefore the outcome has 247 some potential to be impacted by error and/or bias, no matter how 248 small or tolerable. Jayaprakash [19] highlights that miscarriages 249 of justice are typically a major consequence of human and system errors 250 and therefore professionals should focus on addressing and minimising 251 these issues rather than undermining the concept of individuality 252 and uniqueness (Section 3.1), which is inherent in forensic firearm 253 identification. However, experts should continue to deepen current un- 254 derstanding, demonstrate the scientific foundations of firearm identifi- 255 cation, improve the method by which conclusions are drawn and fully 256 acknowledge the limitations and significance of their interpretations 257 when applied to casework (Section 4). The evolving nature of science, 258 also means that as our understanding and knowledge continues to ex- 259 pand it may be inevitable that interpretations made in the past, may 260 upon reflection, be seen as too significant/conclusive or unfortunately 261 proved incorrect. This limitation should not cause growing or emerging 262 disciplines, including forensic firearm identification to be deemed inad- 263 missible in court, just that when conclusions are communicated to lay- 264 persons the capabilities and limitations of the evidence should be 265 appropriate to ensure the correct weighting and significance is assigned 266 in the decision making process.

\section{The firearm identification process and the firearm examiner}

Modern forensic firearm identification is principally concerned with 269 comparing the surface contours of two components of ammunition cre- 270 ated during the firing process to establish if they could have been fired 271 from the same firearm. In a firearm, a component part (of harder mate- 272 rial) operates as a tool that transfers gross (class characteristics) and 273 fine (individual characteristics) features (toolmarks) to the comparably 274 softer ammunition surface. Toolmarks can be created by either impres- 275 sion (perpendicular compression between two surfaces) and/or stria- 276 tion (impression plus lateral movement between two surfaces; also 277 known as engraved) and the differential importance of class and indi- 278 vidual characteristics in the process of identification are further ex- 279 plained in Section 3.1.

Table 2 summarises the possible range of conclusions that may result 281 from a comparison between similarly fired ammunition components as 282 proposed by The Association of Firearms and Tool Mark Examiners 283 (AFTE). Conclusions are achieved by observing these characteristic sur- 284 face features using a standard examination method for firearm identifi- 285 cation outlined by the Scientific Working Group for Firearms and 286 Toolmarks (SWGGUN [20]), which comprises of four stages:

1. Evaluation-class characteristics are observed by eye between two 288 specimens; if these agree then the comparison moves into stage 2, 289 if they do not agree then the specimen is eliminated as having 290 come from the same tool.

2. Comparison-comparative examination of the subclass (see AFTE 292 Glossary [21] and/or individual characteristics between specimen 293 through pattern matching using a comparison macroscope (or 294 microscope) 
t2.1 Table 2

t2.2 Definitions for range of conclusions related to the AFTE theory of identification [21].

$\mathrm{t} 2.3$

\begin{tabular}{|c|c|}
\hline Conclusion & Definition \\
\hline Identification & $\begin{array}{l}\text { Agreement of all discernible class characteristics and sufficient agreement of a combination of individual characteristics where the extent of agreement } \\
\text { exceeds that which can occur in the comparison of toolmarks made by different tools and is consistent with the agreement demonstrated by toolmarks } \\
\text { known to have been produced by the same tool. }\end{array}$ \\
\hline Inconclusive & $\begin{array}{l}\text { A. Agreement of all discernible class characteristics and some agreement of individual characteristics, but insufficient for an identification. } \\
\text { B. Agreement of all discernible class characteristics without agreement or disagreement of individual characteristics due to an absence, insufficiency, } \\
\text { or lack of reproducibility. } \\
\text { C. Agreement of all discernible class characteristics and disagreement of individual characteristics, but insufficient for an elimination. }\end{array}$ \\
\hline Elimination & Significant disagreement of discernible class characteristics and/or individual characteristics. \\
\hline Unsuitable & Unsuitable for examination \\
\hline
\end{tabular}

3. Conclusion-determination if there is sufficient agreement between only the individual characteristics to render one of the ranges of conclusions.

4. Verification-peer-review process to evaluate the conclusions of the first examiner as outlined within a laboratory's quality assurance policy [22].

The AFTE theory of identification [21] is principally a spatial relationship comparison of the reproducible, three dimensional (width, height/ depth, curvature) unique and individual surface contours within two toolmarks to determine whether they have been produced by a common source (tool). Moran [23] provides an overview to the changes and development of the theory of identification as it relates to toolmarks, a theory that has been reviewed and clarified repeatedly since the 1980s and should continue to be studied and researched. If there is sufficient agreement in class and individual characteristics this is commonly referred to as a match, however, do not misinterpret the term 'match' as the two toolmarks being $100 \%$ identical (Section 3.1). To contextualise how the range of firearm identification conclusions can provide corroborative evidence and intelligence to an investigation, Table 3 summarises the capabilities and limitations of firearm identification.
The science underpinning firearm identification is more complex 316 than some other forensic disciplines due to the wide range of variables 317 that require consideration in the production of toolmarks used in the 318 comparison and identification process. The type and material composi- 319 tion of both firearm and ammunition used, the quality and consistency 320 of the manufacturing processes, the history of the weapon and ammuni- 321 tion since production and even the environmental testing parameters 322 may all influence the ability of firearm components (i.e. tools) to create 323 reproducible toolmarks on the surfaces of fired ammunition. It is be- 324 yond the scope of this article to explain the scientific mechanisms relat- 325 ed to each of these variables and research cannot be expected to be 326 publishable for every ammunition-manufacturer combination that 327 may ever exist when many thousands of firearm models and ammuni- 328 tion lines are in existence. Therefore, examiners may be required to in- 329 vestigate, test and evaluate the evidence on a case-by-case basis and this 330 process is substantially more difficult when the firearm is not recovered. 331 Unfortunately, a 'no gun case' is a common occurrence in shooting in- 332 vestigations worldwide as criminals have become more aware of the ca- 333 pabilities and limitations of forensic science to investigate crime. No gun 334 cases are often only used for intelligence purposes so the implication of 335 errors are less significant than cases involving evidential analysis and 336

Table 3

Comparison between the capabilities and limitations of forensic firearms identification for the AFTE range of conclusions.

\begin{tabular}{|c|c|c|}
\hline Conclusion & Capabilities & Limitations \\
\hline \multirow[t]{4}{*}{ Identification } & $\begin{array}{l}\text { Unique identification of suspect weapon to discharging the fired } \\
\text { ammunition components using individual characteristics }\end{array}$ & $\begin{array}{l}\text { The identification of fired ammunition components from a crime scene can } \\
\text { only be identified to a firearm when a weapon is recovered for test-fire } \\
\text { comparison }\end{array}$ \\
\hline & $\begin{array}{l}\text { Comparison between multiple corresponding fired exhibits can determine } \\
\text { the type and number of weapons that were discharged at the crime scene } \\
\text { when no weapon is recovered for test-fire comparison (inferred weapons) }\end{array}$ & $\begin{array}{l}\text { Identifications cannot routinely be made between the abraded surfaces of } \\
\text { shot fired simultaneously through a smooth bore shotgun barrel as individual } \\
\text { characteristics in abrasions are not reproducible }\end{array}$ \\
\hline & $\begin{array}{l}\text { Comparison between corresponding fired exhibits from multiple crime } \\
\text { scenes can identify a crime series by linking to an inferred weapon }\end{array}$ & $\begin{array}{l}\text { Cannot determine who fired the weapon, when the weapon was fired or how } \\
\text { long fired ammunition components or firearm has been in the location of a } \\
\text { crime scene }\end{array}$ \\
\hline & $\begin{array}{l}\text { Identification and comparison of reproducible individual characteristics on } \\
\text { the surface of plastic wadding fired through a smooth bore shotgun barrel }\end{array}$ & $\begin{array}{l}\text { It is not always possible to determine the make and/or model(s) of the guns } \\
\text { used to fire ammunition components recovered from a crime scene }\end{array}$ \\
\hline
\end{tabular}

Inconclusive $\quad$ Some evidence to support the suspect weapon may have fired the Some evidence to support the suspect weapon may have fired the
ammunition components and insufficient evidence to eliminate

Elimination Elimination of suspect weapons from discharging the fired ammunition components using class characteristics

Insufficient individual characteristics to support identification to a specific weapon

In cases where the weapon was recovered at some point after the crime occurred, eliminations based on comparison of individual characteristics to test-fired samples may be incorrect if there was opportunity for the component surface to naturally change and wear so that identification may no longer be possible (note, in most cases examiners are more likely to render an inconclusive conclusion than an elimination, especially if damage appears to be deliberate in nature)

Firearms evidence cannot be used to eliminate or identify the firearm that may have discharged it

Firearms evidence lacks firearm produced toolmarks e.g. lead core, lead fragment or ogive portion of a bullet 
interpretation that may reach court. The interpretive approach utilised in casework (e.g. Bayesian; see Section 4.3) may then be chosen depending on the nature (intelligence or evidential basis) of the information attained.

Most firearm-ammunition combinations demonstrate reproducible toolmarks for comparison when consecutive test-fires are produced. The number of test-fires may be two or three, or another arbitrary number determined only through examination and testing. Variability in reproducibility of toolmarks may be due to the condition of the firearm and/or ammunition surface being utilised when the toolmark is created. To produce a repeatable pattern for comparison it is important that all the possible variables are consistent as possible. Reference test-fires from suspect weapons for comparison to evidence samples therefore should fire where at all possible, the same type and specification of ammunition. If the manufacturing consistency and tolerance of the ammunition (including cartridge dimensions, weight of bullet, composition and quantity of combustible propellant etc.) is poor this may adversely affect the quality and reproducibility of the toolmark created on each cartridge of ammunition fired and hence more than three test-fires may need to be created to undertake the comparison.

Heard [7] theorises a scenario where only 20 individual characteristics (striations) match in a firearm identification comparison and calculates that the chance of another tool creating a toolmark with these same matching striations is 1 in $193,730,707,456$. Such probabilities are considered by AFTE as a practical impossibility [21], although this statistical model has not yet been applied to a real case for evaluation and review. Typically, as long as the firearm component such as a barrel has not been damaged by corrosion or some other external influence, for example cleaning with a steel rod or heavy use of steel wool then a fired bullet is likely to have sufficient agreement to match the 100th fired component to the 1st. Research has demonstrated with firearm components that are particularly resistant to wear that the 5000th [24] and even 10,000th [25] test-fired component can be matched to the 1st. Research using traditional comparison methods have also been supported by quantitative comparisons for 500 consecutively fired cartridge cases [26]. However, in other types of firearms or when firing different ammunition the variation in individual characteristics may be sufficient to make identification questionable after only 50 fired cartridges [27]. There is also no guarantee that a firearm will produce usefully reproducible marks from one shot to the next, meaning that not all fired ammunition components may be able to be identified to the original firearm source. Such variation in the degree of degradation in toolmark quality and reproducibility will in part depend on the materials and manner in which the weapons are used and the resulting wear of the firearm components. Table 4 illustrates how a number of factors may affect the rate of component wear. Firearm examiners should therefore consider undertaking such investigative comparisons as part of their training and/or continuous professional development to assess the extent of variability in individual characteristics, especially when casework questions the use of a firearm since the crime occurred.

Laypersons cannot be expected to know the influence all these variables that may need to be considered and referred to when making their interpretations; hence why an expert is required. A body of published research [28-30] has been compiled identifying some key research in the field using not only the traditional 'more subjective' 391 approach, but also employing a variety of empirical, more 'objective' ap- 392 proaches (Section 3.2). All the studies to date have ultimately supported 393 the theory of identification, however, for those working outside the dis- 394 cipline it is not correct to assume that firearm examiners believe this 395 body of published work is sufficient. As part of the examiner's role, re- 396 search is frequently conducted to test scientific theory and hypothesis 397 related to particular casework scenarios using appropriate scientific 398 methods. Such research is especially important when encountering 399 casework where examiners observe new or unexpected phenomenon 400 or features that require more background investigation before conclu- 401 sions can be drawn. Due to case load and prioritisation of undertaking 402 casework, unfortunately much of the practice-led research does not 403 necessarily get disseminated through formal written publication. Key 404 information does get disseminated through other mechanisms, such 405 as verbal communication in annual training seminars, conferences and 406 practitioner forums, although this dissemination method will not be 407 as extensive.

Regrettably, rigorous scientific method and quality assurance pro- 409 cesses have not always been employed by all firearm examiners over 410 the discipline's history and this has been demonstrated by emerging 411 miscarriages of justice. Unfortunately it is likely that these cases will 412 still continue to emerge from the past. However, due to improvements 413 in procedure, accreditation and training (Sections 3.3 to 3.5) since 414 2010 , recent case outcomes are hopefully less likely to be questionable. 415 To reduce erroneous evidence being admitted into the courtroom, the 416 author advises laypersons not to make assumptions on the work under- 417 taken by an expert and to promote dialogue between legal counsel and 418 expert witnesses at the earliest opportunity.

The NAS [2] study had a significant impact and has encouraged prac- 420 titioners and academics to actively increase the number of research 421 publications specific to the field of firearms and toolmark identification. 422 Fully qualified and experienced examiners are being motivated to pur- 423 sue self-funded research-based Masters and PhD programmes in foren- 424 sic firearm identification in their own time for example, to increase 425 publication and research outputs in the field. The author believes pub- 426 lished research outputs will continue to increase over time, especially 427 due to the relatively recent growth in number of collaborative projects 428 and committees involving key stakeholders of both practitioners and 429 academics within the field.

\subsection{Class and individual characteristics}

Firearms are designed to discharge specific types of ammunition and 432 typically each model of firearm produced will exhibit the same gross 433 features known as class characteristics in every firearm produced with 434 this design. In barrels for example, gross features include the calibre or 435 gauge, number of lands and grooves, direction and angle of rifling 436 twist, groove profile and groove depth, which will all be similar. All of 437 these features i.e. class characteristics are transferred through an en- 438 graving action to the surface of the projectile (e.g. bullet) as it travels 439 down the barrel towards the muzzle (barrel exit). For any component 440 part that comes into contact with ammunition there are designated 441 class characteristics (Fig. 1) to describe and compare the dimensions, 442

Table 4

Some factors potentially affecting the speed of change of original manufacturing toolmarks on firearm components.

\begin{tabular}{|c|c|c|}
\hline Factor affecting wear & Example of how the factor could affect wear & Potential influence on speed of toolmark change on component surface \\
\hline Firearm component material properties & Increased hardness and density of material e.g. gun steel & Reduces rate of toolmark change by increasing resistance to wear \\
\hline Manufacturing processes & $\begin{array}{l}\text { Hammer forging or swaging compresses the material } \\
\text { increasing density and hardness }\end{array}$ & Reduces rate of toolmark change by increasing resistance to wear \\
\hline Use of the weapon & $\begin{array}{l}\text { Fast consecutive firing causes accumulation and increase } \\
\text { in temperature inside weapon }\end{array}$ & Increases wear and rate of toolmark change \\
\hline Cleaning method of weapon & Use of abrasive cleaning materials & Increases the degree of toolmark change \\
\hline Deliberate damage & $\begin{array}{l}\text { Corrosion due to water; damage due to extreme } \\
\text { temperature (fire); damage from tools }\end{array}$ & Increases the degree of toolmark change \\
\hline
\end{tabular}




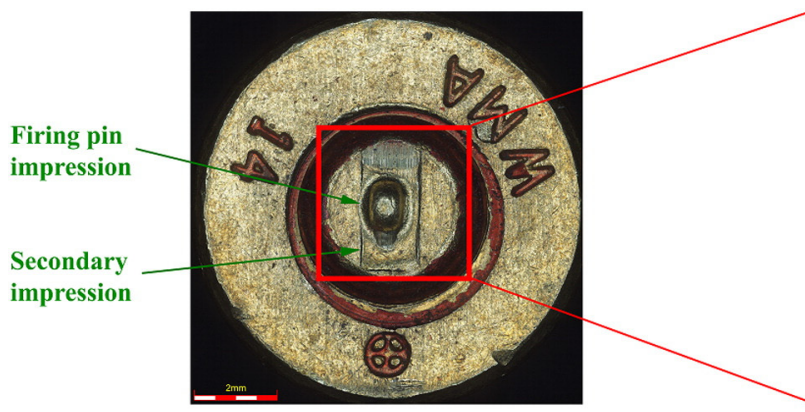

a)

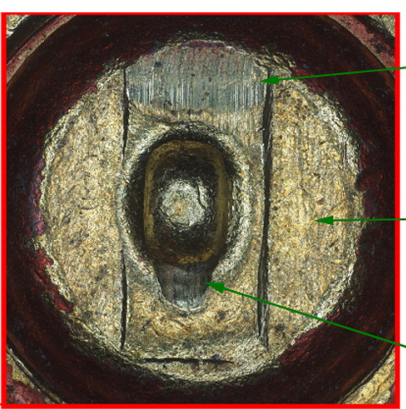

b)

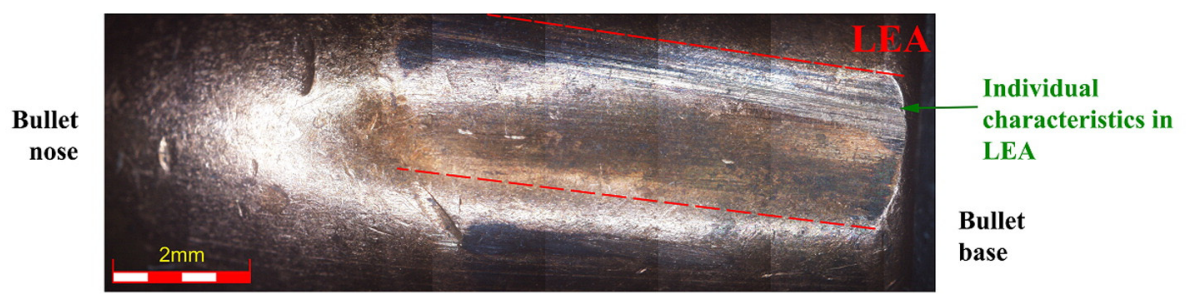

c)

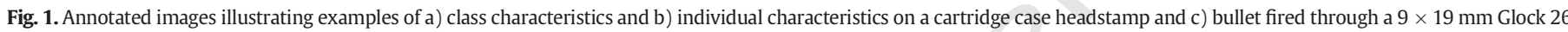
semi-automatic pistol.

geometries and relative spatial relationships between these to allow gross differentiation between different firearm models.

The individual characteristics are toolmarks (striations) on the surface of the firearm component that are randomly generated by the natural wear of the manufacturing tool surfaces. The number, width and depth of striations within any toolmark area on a firearm component surface are therefore specific and unique to each component part produced. The toolmarks present on the firearm component surface are then transferred by impression and/or engraving actions to the ammunition surface(s) during the firing process. Over the lifetime of the component the manufacturing toolmarks will be randomly modified to some extent by wear depending on the use of the firearm. As these striations are random, the special pattern and dimensions are unique and thus allow for differentiation between separate firearm components manufactured by the same tool.

This means that two weapons of the same manufacture and model cannot be differentiated by comparing the class characteristics alone. Individualisation and identification can only arise through the comparison of corresponding individual characteristics. The greatest similarity between individual characteristics may be visible when comparing the toolmark surfaces of consecutively manufactured components. However, there is still sufficient uniqueness between the striated toolmarks to differentiate consecutively manufactured surfaces [31]. This may be due to the manner of interaction between the ammunition and firearm component surface and/or the processes involved in modern manufacturing methods. For example, finishing processes may be applied to the surface after cutting or forming the component parts [32], which generates further individualising toolmarks on the surface and thus similarity of striated or impressed toolmarks on consecutively manufactured components is further reduced.

In forensic firearm identification, the transfer process of the manufacturing toolmarks (individual characteristics) on the parent component surface to the fired ammunition surface(s) is an important consideration. The chemical and physical composition of the ammunition surface will affect the degree of transfer of the individualising toolmarks. The individual characteristics observed on the ammunition surface are typically not duplicated exactly from the parent tool surface and are considered as another unique pattern, whilst retaining some of the parent tool's uniqueness. As a result, it is very important to compare like-forlike surfaces where possible when undertaking firearm identification.
As mentioned previously, no-one has yet observed the individual charac- 483 teristics of any two fired ammunition surfaces to be sufficiently similar 484 when fired from different weapons. There have been occurrences 485 where very similar non-class marks have been produced by different 486 guns, but then this is attributed to 'subclass' effects. The potential issue 487 arising here is that there is no clear division of when a striation is 488 categorised as an individual or subclass characteristic. In these instances 489 an examiner should undertake research to determine the potential for 490 subclass to occur using the firearm in question and comment on the ev- 491 idential strength of the conclusion reached.

A comparison between two different toolmarks knowingly created 493 by the same tool will never result in surface contours that are identical 494 in their surface topography. Vanderkolk [33] explains this variation is 495 natural and expected due to the variability in environmental factors in- 496 volved with surface interactions and "noisy randomness" in patterns; 497 patterns that are not permanent and will change, albeit slowly or quick- 498 ly. Thus comparison and 'matching' requires a level of sufficient agree- 499 ment to allow for some degree of natural variation, which can be 500 achieved by both subjective and objective mechanisms (Section 3.2). 501 The term 'match' in comparison science is therefore not identical and 502 should not be expected to be as such unless one compares exactly the 503 same toolmark with itself. The experience and training of a practitioner 504 and use of a scientific method is therefore important to determine the 505 toolmark surface contours that are reproducible and the contours that 506 result due to natural variation in the interaction between a specific 507 tool and surface pairing. Such evaluation is achieved in practice by com- 508 paring multiple test toolmarks after examination of the question evi- 509 dence toolmark surface. Exposure to very similar, but knowingly 510 different toolmarks allows the practitioner to develop what is some- 511 times referred to as a 'best known non-match' that allows a practitioner 512 to evaluate the quantity and quality of individual characteristics in 513 questioned toolmarks encountered during casework. However, exam- 514 iners should remain "conservative when reporting the significance of 515 these observations" [34] when they themselves did not see the specific 516 tool creating the toolmark being observed.

AFTE have categorised the reporting of firearm identification range 518 of conclusions (Table 2) as an identification, elimination or inconclusive 519 with a fourth category stating that the evidence is unsuitable for foren- 520 sic comparison (for example, the exhibit is damaged and does not ex- 521 hibit any class or individual characteristics to allow for a comparison). 522 
Prior to 2012, practitioners in identification based disciplines, including firearm identification, defined the term individualisation to mean the identification of a common source to the exclusion of all others [35], thus reporting an identification in firearms-related casework to the exclusion of all other guns, for example. However, this definition was understandably challenged and forensic identification-based disciplines have since accepted that it is not realistically possible for an examiner or discipline to examine and compare the individual characteristics exhibited by each source (e.g. firearms or tools) in existence within the world. In the case of firearms, it is estimated that there are now well over 875 million firearms in the world [36], therefore sampling within the discipline observed through casework is insufficient to represent truth about individualisation within the entire population. However, due to the naturally random patterns and comparative research undertaken between toolmarks created by consecutively manufactured components, there is support that toolmarks can be differentiated between even within these manufactured components. As a result, there is a high level of probability that toolmarks created by different sources can be differentiated between if the quality and quantity of individual characteristics present in the toolmarks compared is high enough. SWGGUN [30] therefore states that "any individual association or identification conclusion ... is based not on absolute certainty but rather on the practical certainty of the underlying (validated) scientific theory".

Philosophical arguments exist stating there is no such thing as individualisation [37,38], however, Kaye [39] counters some of these statements and highlights that some of the criticisms made of forensic science disciplines are common and inherent in science generally. Chalmers [40] has written a book on the philosophies of what constitutes 'science', but underlying this constant philosophical debate is the concept that 'science' starts out with an observation which takes significant time (sometimes hundreds of years!) and a body of published and critiqued research to support a fundamental 'scientific' principle at any particular moment in time. There is continual movement towards empirical studies to support the hypothesis of general rather than unique individualisation particularly using more objective three-dimensional (3D) imaging and comparison databases (Section 4.2). Saks [41] identifies that whilst the body of available empirical studies is expanded, forensic scientists must be honest about the limitations of the interpretations and information available when applied to casework and the author agrees.

In case of firearm identification, there may be some non-matching striae evident between a test-fired component and its evidence exhibit. Heard [7] states that it is "by experience and training alone that the examiner is able to determine which are relevant and which are nonrelevant microstriae". To evaluate the variability in striae created on fired ammunition components the examiner should visually compare consecutively fired cartridge component from the same firearm under the comparison macroscope, but preferably after examining the crime scene evidence. This could reduce some elements of subjectivity that will be further discussed in the next sub-section.

When comparing unique reproducible striations (striae) on fired bullets and cartridge cases that are known to have been fired from different weapons, some striae will coincidentally align and appear to match from chance alone. However, it has been shown through empirical research that the number of quantitative consecutively matching striations (CMS) observed in two-dimension (no depth considered) is unlikely to exceed a minimum of a single group of eight striations or two groups of five striations [42]. Within every comparison there are likely to be hundred if not thousands of striae that are considered. An examiner should use more than one location on the fired surface; they will compare all forensically significant features available on an exhibit surface to establish not only a level of agreement, but also evaluate any disagreements in the surface contours being compared. Such locations can include multiple consecutive land engraved areas (LEA; Fig. 1) on fired bullets or multiple features on cartridge cases including firing pin impressions, breechface impressions, extractor claw marks etc. Thus when an identification is made, it is usually not the comparison of 589 only one part of the component, but multiple areas and probably arising 590 from multiple firearm components. The probability of the toolmarks 591 therefore being rated as 'identifiable' in all of those locations on a single 592 exhibit under comparison, makes the conclusion of an identification 593 more probable, although still not absolute.

\subsection{Subjectivity, objectivity and bias}

Interpretation and comparison of objects and information includes 596 elements of both subjectivity and objectivity. During an examination 597 and comparison between two objects, the examiner will assess objec- 598 tive measurements of the relative features being observed, however, 599 does not necessarily report these as quantitative data. The examiner 600 uses their training and experience to determine whether individual 601 characteristics under comparison have sufficient agreement for them 602 to be considered as coming from the same source (firearm in this 603 case) or another source. The main criticism in firearm identification is 604 related to the high level of conceived subjectivity associated with pat- 605 tern matching, which is not supported by objective justification. If sub- 606 jectivity was not part of this process then anyone could undertake the 607 analysis and thus prior training and experience would not be required 608 [33]. However, miscarriages of justice have demonstrated the effect of 609 lack of sufficient training, inconsistency in comparison process and is- 610 sues in reporting findings by some firearm examiners. Therefore, efforts 611 are required to increase objectivity and improve consistency in practice 612 and reporting.

Over the last 20 years, more objective methods have been developed 614 and implemented in firearm identification advancing beyond line 615 counting and CMS, quantifying and comparing measureable changes 616 in 3D surface contours by more automated and repeatable mechanisms. 617 Such technological approaches were developed for the scientific field of 618 surface metrology and have been adapted for this specific application. 619 Such high accuracy, high precision techniques are discussed further in 620 Section 4.2 and have been able to provide evidence to objectively sup- 621 port the theory of identification through mathematical comparison al- 622 gorithms. However, this equipment is very expensive, continuously 623 evolving and researchers are yet to optimise the technology for this be- 624 spoke application (Section 4.4). Objective approaches alone are current- 625 ly unable to meet the subjective interpretation accuracy of complex 626 outcomes achieved by trained experts [43]. It is unlikely that the full ca- 627 pability and widespread incorporation of such objective comparison 628 techniques will meet or exceed that of the expert, meaning these tech- 629 nologies will only aim to support the reliability of examiner's interpre- 630 tations. Questions relating to potential bias and human error will 631 therefore remain, but can be significantly reduced.

Dror $[40,42]$ has undertaken research into the effect of various types 633 of bias that can affect the subjective nature of forensic comparison for 634 identification. He has identified that some examiners are more prone 635 to bias than others as well as the fact that comparing incomplete or par- 636 tial patterns has a greater potential to produce variability in the range of 637 conclusions attributed to identification [44]. The work of Dror and 638 others in the psychological sciences has been seen to negatively impact 639 the confidence in forensic comparisons and judges have since excluded 640 forensic evidence from the courtroom due to the potential for bias to 641 lead to erroneous identification. In the majority of routine cases, issues 642 with bias are significantly reduced if the comparison is not complex 643 [45]; complex examples include comparison of fragmented and signifi- 644 cantly damaged evidence. Bias-based research is important to improve 645 the expert's understanding of how examination procedures may intro- 646 duce bias, identify actions that can be taken to reduce the chances of 647 bias occurring and appreciate how bias can potentially adversely affect 648 identifications made during casework [46]. For example, implementing 649 protocol to examine firearms exhibits before test-fired reference sam- 650 ples from suspect weapons. Principally, published research indicates 651 that it is not the science within forensic identification disciplines that 652 
is necessarily 'flawed', but the psychological manner that humans conceive and compare information that has the potential to affect the outcome of comparative pattern recognition.

The protocol within forensic firearm identification should routinely involve the use of independent peer-review by another firearms expert to verify that the conclusion drawn from any comparison is correct. However, at present, the protocol employed varies between laboratories globally with some employing verification only for identifications. Such verification protocol can again lead to the incorporation of confirmation bias [47] as the peer-reviewer already assumes that the comparison has been classified as an identification. As a measure to reduce the potential for confirmation bias, some laboratory protocols require peerreview across the full range of possible initial examiner conclusions (elimination, inconclusive and identification). In addition, to remain independent, peer-reviewers should not be informed of the initial examiner's conclusion. Such necessary changes in protocol are being employed, but may take years to be common practice worldwide as in many routine cases such action may be an un-necessary finance and human resource [48].

The development and use of forensic comparison databases (Section 4.2) have also been introduced to forensic firearm identification, which can reduce bias to some extent. Automatic database comparisons utilise algorithms in computer software to blindly assess objective criteria and quantitative data between samples electronically stored within it resulting in a ranked list of potential matches. However, humans are still required to undertake the final assessment from a short-list of possible matches so randomisation protocol should be employed to reduce bias resulting from ordinal ranking of the samples [46]. Recent research using competence test sets still supports that more accurate comparative conclusions arise from firearm examiners than using the 'more objective' blind algorithm-based comparison method when sample data is stored electronically within a database [43].

Although the author strongly recommends that subjective interpretation in casework is evidenced by objective quantitative data to demonstrate and scientifically justify the interpretation, at this time, there needs to be some compromise between ideal and realistic approaches. Currently, resourcing and capacity limitations experienced by many examiners across the world mean that they may be unable to meet ideal levels of objective reporting in casework and further improvements need to be implemented to make this more achievable for all. Understandably these advances will take considerable time and funding to ascertain and implementation is typically beyond the control of firearm examiners, but lies with government offices and policy makers [49]. As a minimum there has been a move to improve consistency in quality of forensic practice by introducing procedural standards and laboratory accreditation (Section 3.3) as well as increased requirements for proficiency and competency testing (Section 3.4). However, it should not be acceptable for laboratories to ignore recommendations supported by research from other scientific disciplines.

\subsection{Procedural standards and accreditation}

The 21st century has seen significant changes in the expectations of many laboratories and their employees to undertake procedures according to national (e.g. Laboratory Accreditation Board of the American Association of Crime Laboratory Directors [ASCLD/LAB]) and international standards of practice (e.g. International Organisation for Standardisation [ISO] 17025 and 17020 for laboratories and crime scene units respectively). The United Kingdom Accreditation Service (UKAS) is a profit based organisation that accredits against ISO, whereas ASCLD/LAB is non-for profit and seeks to provide an enhanced ISO 17025 standards in their accreditation programme. The costs associated with the assessment of such accreditation can be very high and thus not all regions have the finances to acquire such recognition. In addition, conforming to such standards can limit the use of available equipment for research and non-standard (or new) methods of testing and analysis, which may stifle the progress of in-house research in forensic 717 disciplines by practitioners internationally. If a unit/laboratory does 718 not have ISO accreditation this does not mean it is not operating to ap- 719 propriate standards and practice, just that it has not yet been assessed to 720 be. The policies for employing standardisation of practice vary from 721 country to country, but there are improvements and developments 722 being introduced every year to improve this. In the UK, the Forensic 723 Science Regulator stipulated in the Codes of Practice and Conduct [50] 724 that all firearms and toolmark based examination and analysis should 725 be undertaken in ISO 17025 accredited laboratories by April 2012. 726 Until a body or government places compulsory requirements on all lab- 727 oratories/units to acquire such accreditation then this will not be sought 728 by all.

To encourage and facilitate dissemination of best practice between 730 practitioners US governments have funded Scientific Working Groups 731 (SWG) in various forensic disciplines e.g. SWGGUN. In 2014, SWGs 732 were replaced by the Organization of Scientific Area Committees 733 (OSAC) to more effectively strengthen the scientific basis of forensic 734 science disciplines in the US as well as create consistent documentary 735 standards and guidelines for dissemination within each discipline. The 736 initiative was established by National Institute of Standards (NIST) 737 and Department of Justice (DOJ) and the OSAC community, comprising 738 of 24 sub-committees [51], is comprised of both experienced practi- 739 tioners and relevant professionals from agencies, academia and indus- 740 try. The two relevant sub-committees for the firearm discipline are 741 firearms and toolmarks and gunshot residue.

Accreditation and best practice alone is not sufficient to ensure the 743 accuracy of the interpretation of casework undertaken by examiners, 744 however, UKAS for example, assesses and audits the laboratory or de- 745 partment as a whole to ensure that employees follow the laboratory's 746 standard operating processes and procedures. However, UKAS does 747 not assess individuals specifically to determine whether they carry out 748 the procedures and interpret results competently. This is where qualifi- 749 cations (Section 3.4), training and additional and regular testing of indi- 750 viduals (Section 3.5) are required.

\subsection{Higher education qualifications}

Employment of firearm examiners with higher education based aca- 753 demic qualifications has been increasing over the last 50 years. The 754 greatest change in provision of such qualifications in forensic science 755 has been since late 1990s where public interest in forensic science in- 756 creased and higher education institutes (universities) began offering 757 degrees in areas of forensic science, dramatically raising competition 758 for forensic-related jobs. In the field of firearms and ballistics, this 759 change has resulted in the employment of an increasing proportion of 760 entry level technicians and trainee examiners with science degrees (at 761 undergraduate and postgraduate level) thus demonstrating a deeper 762 initial scientific understanding and having some prior experience in un- 763 dertaking forensically significant research prior to starting work. Inter- 764 estingly there has also been a significant rise in the number of 765 qualified female firearm examiners being employed in this discipline 766 over the last 10 years.

767

Heard [7] mentions that there are two best professional qualifica- 768 tions currently offered in the Firearms and Toolmark Examination 769 which involve no 'taught' elements and rely solely on the candidate's 770 (practitioner's) prior training and casework experience in the relevant 771 discipline. Only one is an academic qualification; the UK's Forensic Sci- 772 ence Society (now the Chartered Society of Forensic Sciences [CSFS]) 773 Professional Postgraduate Diploma (Strathclyde University) with an op- 774 tional Masters level research-based Top-Up Qualification (Staffordshire 775 University). The other programme is offered by AFTE and is discussed 776 further in Section 3.5.

Due to the increasing expectation and scrutiny of outcomes from 778 the criminal justice system and the NAS [2] report, very experienced 779 practitioners are seeking further research-based and practice-based 780 
qualifications in areas of firearms and ballistics to deepen their knowledge and provide new evidence to evaluate and validate the science underpinning this field. Dr James Hamby and the author have been awarded their research PhDs in Forensic Firearm Identification [52,53]. There are now other experienced firearms practitioners pursuing their PhDs specifically focussing on publishing novel research and advanced validation studies to support challenging casework in the firearms arena. In addition, there has been increasing interest from practitioners to collaborate with academic institutions to advance and expand research in this field that is not linked to academic qualification.

\subsection{Examiner training and competence}

Globally, firearm examiner training is typically undertaken by bringing in external qualified experts to teach courses in the various areas of firearm examination and identification with in-house support through mentoring. There has been some move to create agency funded national programmes, such as the Bureau of Alcohol, Tobacco, Firearms and Explosives (ATF) funded National Firearm Examiner Academy to try to ensure depth and consistency of training, but they only have funding and capability to train approximately 10 new examiners each year. In addition, a basic electronic training programme was published in 2008 by the National Forensic Science Technology Centre (NFSTC [54]) with National Institute of Justice (NIJ) funding, but this requires continual input and mentoring from a trained examiner to support the training process.

So why is training in firearm identification so important to identify a comparative match? A match is only identified when the number of striations that are dimensionally and spatially comparable exceeds those observed in a best known non-match. The best known nonmatch is primarily built up during the examiner's training period, but will continue to develop over their career. Training initially involves the examiner comparing the individual characteristics on the surfaces of numerous fired ammunition components from the same known firearm (known match). The individual characteristics on fired samples are then compared to those test-fired from as many other firearms of the same manufacturer and model as well as other manufacturer-model combinations (known non-matches).

The closest non-match comparisons of individual characteristics will typically arise from test-firings generated from consecutively manufactured firearm components. Such components are manufactured with the same tool surface and thus may exhibit the highest chance of subclass characteristics. Subclass characteristics may be confused as individual characteristics as they are striae that are typically very deep and therefore may carry over from one manufactured surface to another. As a result, these striae are not individual to one firearm component and may have the potential to interfere with comparison and identification. However, just because a manufactured component may exhibit subclass characteristics, that does not mean that these toolmarks are transferred to the surface of fired ammunition, especially if the toolmarks are striated and not impressed.

To determine a best known non-match, the examiner is required to assess the natural test-fire to test-fire variability of known matches, differentiate individual characteristics from any potential subclass characteristics in known non-matches and thus identify that the fired sample has come from one specific weapon rather than another. The best known non-match therefore is established when the individual characteristics are sufficiently similar that they could be incorrectly identified as being from the same source without sufficient training and experience, but it is known that the individual characteristics produced have been created by firing from two different sources. Objective computerised methods of examination are unable to identify striae as being subclass or individual in nature, therefore subjective human opinion will always be required to interpret this potential during matching.

The number of comparisons that an examiner needs to undertake to determine a best known non-match is a non-quantifiable amount. The degree of individual characteristic similarity between two sources 845 may vary depending on the manufacturing production of the firearm 846 surface and the type of ammunition being fired. It also depends on the 847 training requirements of the institution and the types of firearms typi- 848 cally encountered during casework in that region. The range of firearms 849 and ammunition (including modern and historic) encountered in case- 850 work between regions is likely to differ significantly due to availability, 851 legislation, cost and intended usage. With the vast numbers of manufac- 852 turer-model combinations worldwide it is unrealistic for an examiner 853 to be able to compare test-fires from all of these, therefore the organisa- 854 tion may initially focus training on the manufacturer-model combina- 855 tions that examiners will see routinely in casework, whilst ensuring 856 that all types of firearm and a range of manufacturing methods and am- 857 munition cartridges are observed and compared. The time spent under- 858 taking such comparisons to build up an extensive catalogue of known 859 match and non-match comparisons is typically why firearm examiner 860 training usually takes a minimum of 2 years. Examiners should there- 861 fore appreciate the variables that influence quality and reproducibility 862 and what constitutes a best known non-match to enable firearm identi- 863 fication. Without such training using appropriate scientific operating 864 processes and good working practices it is likely that an individual 865 would not be deemed competent to undertake casework unsupervised. 866

Lack of quality training and use of sub-standard practice has resulted 867 in misidentifications and miscarriages of justice. The recommended way 868 to demonstrate, monitor and rigorously test individual examiners' skills 869 is through the use of competency testing that simulates casework, usu- 870 ally at additional cost to employers and/or employees. Currently, there 871 is no compulsory requirement for examiners to undertake competence 872 assessments or be retested within a specific timeframe [55]. Some dem- 873 onstration of assessment of staff proficiency and competency is recom- 874 mended and usually required within standard operating processes to 875 achieve accreditation, but this assessment could be internal and/or ex- 876 ternal. Ideally examiners should be undertaking annual competence- 877 based assessments that are more robust than proficiency assessments 878 and are assessed by both internal and external organisations to ensure 879 independence from the results.

One externally assessed method demonstrating both written and 881 practical competence is offered by AFTE through their NSFTC assessed 882 certification programme in the examination and identification of three 883 areas; toolmarks evidence, firearms evidence and gunshot residue 884 (GSR) evidence. A practitioner is only eligible to take the test if they 885 are a regular AFTE member or higher, have a total of 5 years of experi- 886 ence in the area of certification and from 2014, also have an undergrad- 887 uate degree [56]. Post-nominal letters of TM, FA and/or GSR-AFTE 888 illustrate an individual successfully completing the AFTE Certification 889 in each of the disciplines respectively. Successful candidates are listed 890 on the AFTE certified members list [57] and the individual must be re- 891 certified every 5 years. A limitation to this programme is that elements 892 of assessment are undertaken in the US, so for international practi- 893 tioners this option is not typically affordable. Also, there are additional 894 competencies undertaken by firearms professionals that are not cur- 895 rently offered through this programme. Other proficiency tests are 896 available from independent companies, such as collaborative testing 897 services providing ISO 17043 accredited tests, that try to fill some of 898 this gap and will ship internationally; however, these still do not cover 899 all key competencies of firearms professionals.

900

Internationally, forensic science provision has seen increasing levels 901 of privatisation resulting in an increasing number of independent prac- 902 titioners or smaller laboratories operating across a range of forensic dis- 903 ciplines. These practitioners or laboratories are not able to develop 904 regular internal proficiency or competency testing and therefore seek 905 sources of external testing. Such privatisation occurred in the UK in 906 2012 with the demise of the Forensic Science Service (FSS). As a result, 907 there was an increasing need for the UK-based professional body, The 908 Chartered Society of Forensic Sciences (CSFS; formerly the Forensic 909 Science Society) to provide external competency testing in a range of 910 
skills undertaken by practitioners such as firearms professionals that was affordable to those working in the UK and covered relevant competencies in their discipline. The Society is an international professional body dedicated to forensic practitioners in all disciplines with members in more than 60 countries. In 2014, the Society was awarded a Royal Charter demonstrating the organisation's importance, constancy and longevity working in this particular field [58]. Consequently, the Society can now provide experienced practitioners the Chartered title of CSFS membership, which demonstrates an individual's high level of professionalism and conduct in their discipline. As a route to becoming a Chartered practitioner in a firearms related discipline, from 2015 UK practitioners can sit Certificates of Professional Competence (CoPC) in legal classification, firearms function testing, comparison microscopy and/or GSR analysis using SEM/EDX microscopy to demonstrate practical competence in court. Practitioner members with either the Accredited or Chartered status are also listed on the CSFS Register of Accredited Forensic Practitioners [59].

In 2014, UK practitioners also highlighted the need to bridge the gap between education and training and therefore the CSFS have worked with forensic practitioners and academics to create the PreEmployment Assessment of Competence (PEAC). The PEAC is a competence based written and practical assessment in crime scene and/or laboratory skills to demonstrate day-one competence of individuals seeking to work in the respective fields. The PEAC aims to act as an independent assessment centre to allow employers to assess the competence and potential of a perspective employee prior to employment and commencement of further training. The first PEAC assessments commenced in June 2015 and the outcome of evaluation of this programme will be published at a later date.

The organisations highlighted in this article, including AFTE, OSAC and CSFS are only some examples of those dedicated to continuing to develop the standards of practice and competence of practitioners and professionals working in the firearms and toolmark discipline. Recommendations and requirements of practitioners and their employers are continuing to develop each year and the author is sure that confidence in the use of forensic evidence analysis in court will be re-established with continuing collaboration and research, dissemination and standardisation of good working practices and developing methods of reporting (Section 4).

\section{Advances in firearm identification}

One of the frequent misperceptions regarding research in forensic disciplines is that research is not undertaken frequently. Within the field of forensic firearm identification, AFTE published a number of articles $[60,61]$ to compile and highlight research undertaken in the field as one way to begin addressing the criticisms highlighted in the NAS report and National Research Council (NRC) report [1] respectively. In addition, Mattijssen [62] reviews the latest research across the discipline between 2010 and 2013 and the SWGGUN Admissibility Resource Kit [30] continues to be populated by AFTE members.

Investigation and research is typically a key component of undertaking casework. In the UK, for example, the former government funded FSS had a research and development budget to undertake novel studies in forensic departments. In comparison to a researcher or academic, publishing research is not identified as a key role for practitioners. Due to high caseloads and other priorities, results, analysis and outcomes from scientific investigations frequently undertaken by practitioners are typically not externally published. At times, it may also be important not to disclose research outcomes into the wider public domain. In addition, articles may be published in a wide range of scientific journals due to the interdisciplinary nature of research in this field and different target audiences for practitioners and academics. Fragmented dissemination of the literature typically makes research inaccessible for practitioners. The most accessible source of literature for practitioners is available through the AFTE journal, however, some criticise the journal's peer-review process due to variability in depth and stan- 975 dard of scientific reporting within some of the articles published. $\quad 976$

To some extent the NAS [2] report has had a positive impact on prac- 977 titioners' opinions regarding the value and importance of publishing 978 their research. There has been some noticeable increase in the encour- 979 agement to support research in some agencies and laboratories to fur- 980 ther build the body of knowledge and evidence underpinning pattern 981 recognition-based disciplines. There have also been increased levels of 982 engagement between academia and industry over recent years to un- 983 dertake collaborative research and share knowledge between scientific 984 disciplines. However, significant human and financial resources are re- 985 quired to be successful in further increasing research outputs in forensic 986 disciplines and to ultimately establish these fields as 'scientific' in the 987 minds of critics. The principal areas of continuing and expanding re- 988 search related to firearm identification focus on undertaking validation 989 studies to provide error rates (Section 4.1), utilising 3D surface metrol- 990 ogy systems and bespoke mathematical automated comparison 991 algorithms (Section 4.2) and incorporating Bayesian statistics into 992 reporting to support evidential findings (Section 4.3).

\subsection{Validation studies and error rates}

994

As previously discussed, there is appropriate research published 995 using both subjective and objective methods to support the theory of 996 identification. Bunch et al. [63] therefore identify that the relevant ques- 997 tion for the courts and the science relating to firearm identification is 998 not whether toolmarks are unique or not, but "Can a trained human 999 or machine reliably distinguish between toolmarks made by one tool 1000 versus toolmarks made by other tools?". Such a question can be an- 1001 swered and empirically determined with comprehensive and blind val- 1002 idation studies to quantify the potential for incorrect conclusions (error) 1003 using quality assured samples created from known sources. To confi- 1004 dently estimate error rates within casework, those sitting the test 1005 must be fully qualified and complete testing blind with limited context 1006 regarding the aim of the test to reduce bias in their conclusions. Practi- 1007 tioner testing should also reflect casework where no prior assumptions 1008 are made about the samples under comparison. In addition, test makers 1009 should not know which test set has been sent to which candidates (dou- 1010 ble blind) and should design the test to reduce confirmation bias typi- 1011 cally evident in proficiency tests when the candidate may have come 1012 to assume that all the unknown samples must come from the reference 1013 samples provided in the test. Examples of good practice using appropri- 1014 ate validation study design were employed during research undertaken 1015 by Kerkhoff et al. [64] reporting no misleading conclusions in a limited 1016 test set of 10 cases and Fadul et al. [65] producing an error rate 1017 (where an examiner made a misidentification) of less than $1.2 \% . \quad 1018$

The NAS [2] study made criticism that there are insufficient valida- 1019 tion studies undertaken in the field of firearm identification. Prior to 1020 the NAS report, Nichols [66] summarised some of the work related to 1021 validation that had been undertaken to this date using the traditional 1022 comparison macroscopy approach. Since then, further research has 1023 been funded, especially in the US, to increase the extensiveness of vali- 1024 dation studies in the field [65] together with more objective validation 1025 studies utilising 3D imaging methods [67,68] from samples fired 1026 through consecutively manufactured components. The outcome from 1027 these studies continues to support the theory of identification and 1028 uniqueness using individual characteristics to identify toolmarks to a 1029 specific common source i.e. a specific gun. Error rates associated with 1030 each study vary, but are typically less than $4 \%[62,63]$.

The type of manufacturing and surface finishing processes employed 1032 when creating a particular firearm component impact on the quantity 1033 and quality of the toolmarks left on the surface, which can be ultimately 1034 transferred during the cycling and/or firing process. The shallower the 1035 gross features of class characteristics and the finer the individual stria- 1036 tions, the more difficult the comparison and potential for larger error 1037 rates to occur [67]. However, the nature of the examination should 1038 
render a conclusion as inconclusive if the examiner feels that the individual characteristics are insufficient to make an identification.

Certainly over the last 5 years there has been increasing change to the research design and methods have been conducted in firearm identification. Stroman [69] discusses and recommends the most appropriate research designs for use in the field moving forward. The author agrees that further research incorporating comprehensive, declared tests with multiple blind elements within the validation methods and an open design (i.e. unknown samples may not all match known reference samples for comparison) should continue to be undertaken to more sufficiently represent the diversity of factors and levels of complexity seen in firearm identification based casework. Unfortunately, due to the time consuming and potentially expensive nature of research, publications using such recommended research methods will not immediately become available. The single blind research publications currently available still have value in supporting the ability of examiners to correctly undertake casework, although the limitations in the research design need consideration when used. In the author's opinion there is insufficient published scientific research to generically rule firearm identification evidence as inadmissible in court.

\subsection{D imaging, databases and comparison algorithms}

Since 1980s databases have been researched, developed and implemented for electronically storing, searching and comparing class and individual characteristics important for firearms investigation and identification. The most prevalent examples include the General Rifling Characteristics (GRC) Database and Integrated Ballistics Identification System (IBIS). With increasing technological advancements since the invention of the comparison macroscope and application of the striagraph [70], firearm identification databases such as IBIS have moved away from comparing 2D images of toolmarks on fired ammunition components alone and incorporated non-contact sensors to acquire 3D surface topography data and complex comparison algorithms to automatically compare fired evidence samples. Brinck [71] established that comparison capabilities were significantly enhanced by using a combination of 2D images and 3D surface data within correlation algorithms. NIST have also designed and produced standard reference materials (SRM) from real fired bullets and cartridge cases; SRM 2460 [72] and 2461 [73] respectively, which are used to establish laboratory protocols for gathering 3D data in a quality assured manner that meet ISO 17025 requirements [74].

Bespoke firearm identification comparison software has been written for specific 3D data formats generated by imaging with different surface metrology technologies [75]. IBIS Trax3D, EvoFinder and ALIAS are examples of commercially available 3D comparison ballistics imaging systems that provide firearm examiners with increased examination objectivity and time-efficiency compared to using a comparison macroscope alone. Surface features can be orientated and contextualised in a wider field of view, depth profiles of contours under evaluation are objectively compared with numerical data and software may allow virtual exaggeration and magnification smaller features of interest. However, comparison algorithms cannot automatically determine whether the samples compared have been generated by the same source, they can only evaluate the similarity of the surfaces compared. Human interpretation is required to make the final comparative conclusion, as previously discussed.

Some laboratory protocols use firearm identification systems as a screening tool to improve workflow efficiency. Representative samples of test-fires and evidence samples may be routinely imaged by trained technicians and stored in the laboratory's database for past, present and future comparison. When comparisons are run during the screening process, the firearm examiner may only review the top 10 or 20 closest database entries, but a confirmed identification can only be achieved when the examiner physically compares the samples under the comparison macroscope. Use of screening protocols may result in missed opportunities by only examining the top 10 or 20 comparisons. How- 1103 ever, limitations arising from missed opportunities usually negatively 1104 impacts current investigations rather than leading to miscarriages of 1105 justice.

1106

The success in solving gun crime and implementing such expensive 1107 objective systems in casework is not down to using technology alone 1108 [49]. Organisations such as ATF, Federal Bureau of Investigation, Interpol 1109 and National Ballistics Intelligence Service (based in the UK) utilise IBIS 1110 for example to share and compare data and intelligence both in their 1111 laboratories and even internationally through Integrated Ballistics Infor- 1112 mation Networks (IBIN). King et al. [76] has evaluated the use of IBIN in 1113 the US demonstrating variable results in effectiveness depending on 1114 how the system was integrated into investigation and casework proto- 1115 cols. Although, not all countries or regions will deem searching of inter- 1116 national databases a routine requirement [77]; more targeted searches 1117 based on intelligence are currently recommended in Europe for exam- 1118 ple. The NRC [1] publication focusses in detail on the development and 1119 potential for forensic ballistics databases although the value of adopting 1120 shared databases is likely to remain debated for some time whilst inter- 1121 agency policies are adapted, implemented and evaluated in each region. 1122 Objective and automated advancements in imaging and comparison 1123 will hopefully further reduce potential for human error resulting in mis- 1124 identifications, but examiner training, experience and competence will 1125 continue to be fundamental, especially as the majority of laboratories 1126 do not have the financial resources to implement such technology. $\quad 1127$

\subsection{Statistical reporting to support evidential findings}

When fired cartridge cases and/or projectiles are recovered from 1129 scenes and there are insufficient individual characteristics to permit 1130 the conclusion of identification, then inconclusive is likely to be report- 1131 ed. However, an inconclusive conclusion has not lost all its probative 1132 value to a case. The likelihood ratio (one element of Bayes' rule) can 1133 be used to measure the relative strength of support for two opposing 1134 propositions considered by the examiner in casework; a suspect weap- 1135 on or another unknown weapon as being responsible for firing the evi- 1136 dence exhibits. For example, Bunch and Wevers [78] use class 1137 characteristics of the firearms evidence and known frequencies of rifling 1138 characteristics in the GRC database to estimate the likelihood ratio and 1139 determine which end of the 'inconclusive' range the evidence is more 1140 likely to support; more inclusive or eliminatory. However, the GRC da- 1141 tabase does not reflect all the possible examples of class characteristics 1142 in existence worldwide. Approximately 4700 combinations were 1143 contained in the 2008 version of the database, but this number is con- 1144 tinuing to increase annually. In addition, the exact number of weapons 1145 that have ever been produced for each manufacturer-model combina- 1146 tion is unknown and neither is the precise number or frequency of 1147 weapons of these characteristics present in the region 'local' to the 1148 crime scene [78]. As a result, any calculation of likelihood in this manner 1149 should utilise an over estimate of the true frequency of weapons in the 1150 population [79] to ensure the strength of the interpretation is not too 1151 strong for the evidence provided. More useful frequency data can be in- 1152 corporated into likelihood ratio calculations by processing information 1153 contained in laboratory case submission records, resulting in more reli- 1154 able and precise estimates of probability assigned to analysis findings. 1155 However, acquisition of such data from records is not typically a routine, 1156 fast or automated process at present and further work needs to be un- 1157 dertaken to facilitate this statistical approach.

New Zealand have been using the calculation of likelihood ratio to 1159 estimate the probability of uncertainty and support their reporting of 1160 interpretations from evidence in court reports for over 20 years across 1161 all forensic science disciplines. The European Network of Forensic Sci- 1162 ence Institutes (ENFSI) have recently published reporting guidelines 1163 [80] to encourage European forensic science laboratories to use likeli- 1164 hood ratio calculations to support their interpretations and the guide- 1165 lines have been adopted for firearms evidence in The Netherlands 1166 
[64], for example. Currently, the US generally appears to be trailing in the routine use of likelihood ratio in firearm identification based casework. Bunch and Wevers [78] discuss the advantages and disadvantages of using the likelihood ratio approach in firearm identification casework for both class and individual characteristics. They conclude with logical scientific support for using the approach above the traditional method so the examiner can utilise all information available to them during the examination. However, the paper highlights the importance for continual training in the use of the likelihood ratio, not only for firearm examiners and legal professionals but also for juries to ensure the approach is understood and utilised correctly in both the laboratory and in court. Research in other disciplines such as fingerprint analysis has also demonstrated that using statistical assessment tools in the examiner's decision making processes did not appear to influence the overall comparison conclusion, although using such tools did improve the accuracy and consistency of the features selected for comparison [81].

\subsection{Continuing developments and research}

Over the last 20 years there have been some significant advancements in knowledge and technology applied within this field, but this is not sufficient and should not stagnate. Research into non-contact 3D imaging technologies and comparison algorithms are continuing to advance objective approaches for firearm identification. NIJ for example are funding Cadre Research Labs to develop a novel gel-based sensor and NIST to create an open-access research focussed resource called the Reference Ballistic Tool Mark Database [82] to enable those without imaging equipment to develop more advanced pattern recognition algorithms. In an ideal world, co-operative polices should be in place to enable all practitioners to access a ballistic identification system to provide objective evaluation and comparison of firearms evidence in casework. Unfortunately implementation of such policies is unlikely to be achievable in the foreseeable future, therefore research into lessexpensive objective methods using traditional comparison macroscopy is also required. Such research could be facilitated through more extensive collaboration between research-based institutions and practitioner laboratories.

Improvements in technology have not only been made in the ability to examine and compare firearms evidence, but also in the manner in which firearm components are manufactured. Advances in modern manufacturing processes to increase both manufacturing tool and firearm component lifetime, accuracy and precision have resulted in finer individual characteristics that are significantly more challenging and time consuming for examiners to compare. Changes in manufacturing approach require particular research attention to evaluate the potential for subclass characteristics and reproducibility of individual characteristics that may influence the ability of examiners to accurately conclude a positive identification during casework. Any research requires funding for both equipment and manpower to improve the perceived reliability of this scientific discipline, however, funding sources worldwide vary significantly and funding available is becoming even more competitive with other forensic disciplines having priority.

Although some countries have sought quality assurance accreditation by implementing standard examination procedures and providing firearm examiner training programmes to improve consistency of practice, these approaches have yet to be employed worldwide. To reduce miscarriages of justice globally these practices need to be shared and individuals should be required to undertake frequent casework simulated competency assessments to demonstrate their ability in drawing correct conclusions for identifications, inconclusives and eliminations. With increased knowledge and scrutiny in the past, present and future judicial processes it is likely that reported incidents of miscarriages of justice will rise from earlier periods where sub-standard practice was employed in casework. It is therefore vital that the field continues to develop and adopt objective reporting practices to support their subjective interpretations through the use of quantifiable methods of comparison and/or statistical models of reporting in casework, such as calculation 1231 of likelihood ratio.

\section{Summary}

Miscarriages of justice arising from firearms misidentification are 1234 typically a result of inadequate standard protocol and/or incorrect inter- 1235 pretation of the firearms evidence rather than 'flaws' in the scientific 1236 underpinning. The practice of firearm identification through pattern 1237 matching is inherently a subjective process and consequently potential- 1238 ly prone to bias and human error, as in many other scientific processes. 1239 However, more objective quantitative methods should continue to be 1240 developed and implemented in casework to support subjective inter- 1241 pretations and improve consistency in reporting of the discrete range 1242 of conclusions. Such approaches include application of statistical 1243 reporting methods and application of non-contact surface measure- 1244 ment techniques.

Research has demonstrated the importance of training in firearm 1246 identification for a number of years before conducting casework unsu- 1247 pervised and regular demonstration of individual competence is recom- 1248 mended. Laboratory accreditation should also be sought to assess 1249 appropriateness and consistency of operational protocol and protocol 1250 should incorporate an independent peer-review process for all conclu- 1251 sions related to casework. In addition, examiners should seek expert 1252 witness training to develop skills in explaining the scientific knowledge 1253 underpinning the discipline and communicating the manner in which 1254 conclusions were obtained to laypersons in court. It should not be ac- 1255 ceptable for an examiners to say 'I am the expert you just have to trust 1256 me' nor laypersons to make assumptions about the work undertaken 1257 by an expert in any field.

Responsibility for evidence admitted into the courtroom resides 1259 with all those involved in the legal process. Law enforcement is respon- 1260 sible for building a case against a suspect by collating intelligence and 1261 utilising expert evidence in the investigative process. The judge should 1262 act as gatekeeper to determine whether a forensic practitioner should 1263 be considered an expert witness and deem the evidence admissible in 1264 the courtroom. Legal counsel should be concerned with asking the 1265 right questions of witnesses both before the court case and during pro- 1266 ceedings to ensure the judge and jury (if relevant) understand and cor- 1267 rectly weigh up the evidence presented to them. Expert witnesses 1268 should ensure that they interpret evidence in a thorough, independent 1269 manner remaining impartial whether they are presenting evidence for 1270 the prosecution or defence.

Hopefully this article has helped to provide a critical review of the 1272 causes of and potential solutions to minimise instances of miscarriages 1273 of justice associated with forensic firearm identification evidence. For 1274 the future, it is recommended that legal professionals seek education 1275 and training from experts in relevant scientific disciplines to become 1276 more aware of the capabilities and limitations of evidence types they 1277 may encounter. Literature written and courses delivered by those with- 1278 out such scientific expertise unfortunately seem to propagate misun- 1279 derstandings and misconceptions within forensic disciplines rather 1280 than aiming to recognise and clarify issues when they arise. The author 1281 therefore wants to request that legal professionals start communica- 1282 tions with subject experts and appointed expert witnesses at the earli- 1283 est opportunity to help them prepare critical and relevant questions 1284 when undertaking cross-examinations. Through two-way correspon- 1285 dence legal counsel can gain further insight into the specific procedures 1286 employed and conclusions drawn in individual cases to develop effi- 1287 cient cross-examination strategies and facilitate effective communica- 1288 tion of the scientific evidence to laypersons in the courtroom. Creating 1289 suitable learning environments in court will inevitably assist the jury 1290 or judge to appropriately evaluate and weigh the evidence presented 1291 to reach a correct verdict in the first instance. If this approach to criminal 1292 proceedings evolves successfully this will ultimately build confidence in 1293 
1294 the scientific reliability of forensic evidence as well as the criminal jus1295 tice system.

\section{Acknowledgements}

1297 The author wishes to thank the following experienced firearms 1298 examiners, Paul Olden (Key Forensics, UK) and Charles Clow (Tarrant 1299 County Medical Examiner's Office, USA) for their time in peer1300 reviewing and proof reading this article prior to journal submission.

\section{References}

1302

1303

1304

1305

1306

1307

1308

1309

1310

1311

1312

1313

1314

1315

1316

1317

1318

1319

1320

1321

1322

1323

1324

1325

1326

1327

1328

1329

1330

1331

1332

1333

1334

1335

1336

1337

1338

1339

1340

1341

1342

1343

1344

1345

1346

1347

1348

1349

1350

1351

1352

1353

Q3 :
[1] National Research Council, Committee to Assess the Feasibility, Accuracy, and Technical Capability of a National Ballistics Database, Daniel Cork, et al., Ballistic Imaging, The National Acadamies Press, Washington, DC, 2008.

[2] Committee on Identifying the Needs of the Forensic Science Community, Committee on Science, Technology and Law Policy and Global Affairs and Committee on Applied and Theoretical Statistics Division on Engineering and Physical Sciences, Strengthening Forensic Science in the United States: A Path Forward, The National Acadamies Press, Washington DC, USA, 2009 (Accessed via <http://books.nap.edu/ openbook.php?record_id=12589\&page $=\mathrm{R} 1>$ )

3] J. Fisher, Forensics Under Fire: Are Bad Science and Dueling Experts Corrupting Criminal Justice? Rutgers University Press, Piscataway, New Jersey, USA, 2008.

[4] ASCLD, ASCLD's Comments on the Release of the NAS Report on Forensic Science The American Society of Crime Laboratory Directors, Garner, North Carolina, USA, 2009

[5] M. Murphy Hong, J.S. Mihalovich, Response Letter From the CAC Following the NAS Report, California Association of Criminalists, California, USA, 2009.

[6] NACDL, NACDL Preliminary Position Statements and Recommendations on Strengthening Forensic Science, National Association of Criminal Defense Lawyers, Washington D.C., USA, 2009.

[7] B.J. Heard, Forensic Ballistics in Court: Interpretation and Presentation of Firearms Evidence, Wiley-Blackwell, Chichester, 2013.

[8] B.J. Heard, Handbook of Firearms and Ballistics: Examining and Interpreting Forensic Evidence, 2nd ed. John Wiley \& Sons Ltd, Chichester, UK, 2008.

[9] M.G. Haag, L.C. Haag, Shooting Incident Reconstruction, 2nd ed. Academic Press, San Diego, 2011.

10] T. Warlow, Firearms, the Law, and Forensic Ballistics, 3rd ed. CRC Press, Boca Raton, USA, 2011.

11] R.E. Walker, Cartridges and Firearms Identification, CRC Press, Boca Raton, 2013.

12] P. Cates, Troy Davis Executed in Georgia Despite Substantial Evidence Pointing to Innocence. Accessed via http://www.innocenceproject.org/news-events exonerations/press-releases/troy-davis-executed-in-georgia-despite-substantialevidence-pointing-to-innocence 2011 (on 24/06/2015).

[13] USA Today, Error-Prone Detroit Crime Lab Shut Down, USA Today. Accessed via http:// www.senate.michigan.gov/sfa/Publications/Notes/2009Notes/NotesJanFeb09bb.pdf 2008, September 25 (on 24/06/2015)

14] B.R. Baker, Detroit Police Crime Lab Closure: Impact on State Police Forensic Science Division Backlog, State Notes: Topics of Legislative Interest January/February, 2009 (Accessed via $<>$ on 24/06/2015).

15] D.S. Bernstein, Bad Ballistics . Accessed via http://www.bostonphoenix.com/boston/ news_features/top/features/documents/05016166.asp October 7-13 2005 (on 24/ 06/2015).

16] S. McVicker, Ballistics Lab Results Questioned in 3 Death Cases. Accessed via http:// www.chron.com/news/houston-texas/article/Ballistics-lab-results-questioned-in3-death-cases-1923892.php March 142005 (on 24/06/2015).

17] D. Dow, M.H. Moon, A Petition for a Writ of Habeas Corpus: Johnnie Bernal vs Douglas Dretke, H-04-1163, 2004.

18] M.E. Olive, W. Long, M.H. Moon, A Petition for a Writ of Habeas Corpus: Nanon McKewn Williams vs Janie Cockrell, 4:02mc00185, 2003.

19] P.T. Jayaprakash, Practical relevance of pattern uniqueness in forensic science Forensic Sci. Int. 231 (1-3) (2013) 403.e1-403.e16.

20] SWGGUN, Firearm and toolmark identification summary of examination methodology, (XXXX). Accessed via <http://www.afte.org/SWGGUN/exammethod.html $>$ on 25/06/2015.

21] AFTE Training and Standardization Committee, Glossary, 6th ed. AFTE, USA, 2013

2] Scientific Working Group for Firearms and Toolmarks (SWGGUN), SWGGUN quality assurance guidelines, AFTE J. 45 (1) (2013) 82-85.

23] B. Moran, A report on the AFTE theory of identification and range of conclusions for tool mark identification and resulting approaches to casework, AFTE J. 34 (2) (2002) 227-235.

24] Y. Ogihara, M. Kubota, M. Sanada, K. Fukuda, T. Uchiyama, J.E. Hamby, Comparison of 5000 consecutively fired bullets and cartridge cases from a 45 caliber M1911A1 pistol, AFTE J. 15 (3) (1983) 127-140.

25] J. Gouwe, J.E. Hamby, S.A. Norris, Comparison of 10,000 consecutively fired cartridge cases from a model 22 Glock .40 S\&W caliber semiautomatic pistol, AFTE J. 40 (1) (2008) 57-63.

26] T.L. Grom, W.E. Demuth, IBIS correlation results of cartridge cases collected over the course of 500 firings from a Glock pistol, AFTE J. 44 (4) (2012) 361-363.

27] M.S. Bonfanti, J. De Kinder, The influence of the use of firearms on their characteristic marks, AFTE J. 31 (3) (1999) 318-323.
[28] R. Nichols, ERRATUM: firearm and tool mark identification: the scientific reliability 1371 and validity of the AFTE theory of identification discussed within the framework 1372 of a study of ten consecutively manufactured extractors, AFTE J. 36 (2) (2004) 124. 1373

[29] R. Nichols, Firearm and tool mark identification: the scientific reliability and validity 1374 of the AFTE theory of identification discussed within the framework of a study of ten 1375 consecutively manufactured extractors, AFTE J. 36 (1) (2004) 67-88. 1376

[30] SWGGUN, Admissibility Resource Kit. Accessed via http://www.afte.org/SWGGUN/ 1377 arkhome.htm 2014 (on 16/03/2015).

[31] A. Zheng, J. Soons, T.V. Vorburger, J. Song, T. Renegar, R. Thompson, Applications of 1379 surface metrology in firearm identification, Surf. Topogr. Metrol. Prop. 2 (014012) 1380 (2014) 1-10 (Accessed via <http://m.iopscience.iop.org/2051-672X/2/1/014012/ 1381 pdf/2051-672X_2_1_014012.pdf 5 on 24/06/2015).

[32] R.S. Bolton-King, J.P.O. Evans, C.L. Smith, J.D. Painter, D.F. Allsop, W.M. Cranton, 1383 Manufacturing of SIG Sauer $9 \times 19$ mm pistols, AFTE J. 44 (1) (2012) 19-28. 1384

[33] J.R. Vanderkolk, Forensic Comparative Science: Qualitative Quantitative Source De- 1385 termination of Unique Impressions, Images, and Objects, Elsevier Academic Press, 1386 London, 2009.

[34] J.E. Murdock, Theory of identification, range of striae comparison reports and mod- 1388 ified glossary definitions-an AFTE criteria for identification committee report, AFTE 1389 J. 22 (3) (1990) 275-279.

1390

[35] Scientific Working Group on Friction Ridge Analysis, Study and Technology 1391 (SWGFAST), Document \#103: Individualization/Identification Position Statement 1392 (Latent/Tenprint). Accessed via http://www.swgfast.org/Comments-Positions/ 1393 130106-Individualization-ID-Position-Statement.pdf 2012 (on 24/06/2015). 1394

[36] A. Karp, Estimating Civilian Owned Firearms, Small Arms Survey: Research Notes 91395 September, 2011.

[37] M.J. Saks, J.J. Koehler, The individualization fallacy in forensic science evidence, 1397 Vanderbilt Law Rev. 61 (1) (2008) 199-219.

38] M. Page, J. Taylor, M. Blenkin, Uniqueness in the forensic identification sciences-fact 1399 or fiction? Forensic Sci. Int. 206 (1-3) (2011) 12-18.

[39] D.H. Kaye, Probability, individualization, and uniqueness in forensic science: listen- 1401 ing to the academies, Brooklyn Law Rev. 75 (4) (2010) 1163-1185.

[40] A.F. Chalmers, What is this Thing Called Science? 4th ed. Open University Press 1403 McGraw-Hill Education, Maidenhead, England, 2013.

[41] M.J. Saks, Forensic identification: from a faith-based "science" to a scientific science, 1405 Forensic Sci. Int. 201 (1-3) (2010) 14-17.

[42] A. A Biasotti, JE. Murdock, Firearms and toolm D.L. Faigman, D.H. Kaye, M.J. Saks, J. Sanders (Eds.),Modern Scientific Evidence: 1408 The Law and Science of Expert Testimony, vol. 2, West Publishing Co., St. Paul, 1409 Minnesota, USA 1997, pp. 131-151.

1410

[43] S. Huang, D. Christophe, The empirical evaluation \& examination of breechfaces on 1411 ten consecutively manufactured pistol slides, 46th Annual Training Seminar, Dallas, 1412 Texas, 24-29 May, 2015.

[44] I.E. Dror, S.A. Cole, The vision in "blind" justice: expert perception, judgment, and 1414 visual cognition in forensic pattern recognition, Psychon. Bull. Rev. 17 (2) (2010) 1415 161-167.

1416

[45] G. Langenburg, C. Champod, P. Wertheim, Testing for potential contextual bias 1417 effects during the verification stage of the ACE-V methodology when conducting 1418 fingerprint comparisons, J. Forensic Sci. 54 (3) (2009) 571-582. 1419

[46] S.M. Kassin, I.E. Dror, J. Kukucka, The forensic confirmation bias: problems, perspec- 1420 tives, and proposed solutions, J. Appl. Res. Mem. Cogn. 2 (1) (2013) 42-52. 1421

[47] D.M. Risinger, M.J. Saks, W.C. Thompson, R. Rosenthal, The Daubert/Kumho implica- 1422 tions of observer effects in forensic science: hidden problems of expectation and 1423 suggestion, Calif. Law Rev. 90 (1) (2002).

[48] C. Champod, J.E. Murdock, D. Howitt, R.M. Thompson, Advances in Image and 1425 Statistical Analysis in Firearms Analysis, Measurement Science and Standards in 1426 Forensic Firearms Analysis, Gaithersburg, USAAccessed via http://www.nist.gov/ 1427 oles/forensics_firearms_2012.cfm 2012 (on 24/06/2015).

[49] P. Gagliardi, The 13 Critical Tasks: An Inside-Out Approach to Solving More Gun 1429 Crime, Forensic Technology WAI, Quebec, Canada, 2010.

50] A. Rennison, Codes of Practice and Conduct for Forensic Science Providers and Practitioners in the Criminal Justice System, Version 2.0, 2014

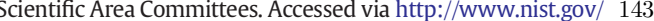
forensics/osac/ 2015 (on 24/06/2015)

[52] R.S. Bolton-King, Classification of Barrel Rifling Transitions for the Identification of 1435 Firearms(PhD) Nottingham Trent University Nottingham, England, 2012.

53] J.E. Hamby, Forensic Firearms Examination(PhD) University of Strathclyde Glasgow, 1437 Scotland, 2001.

[54] The Research, Development, and Evaluation Agency of the U.S. Department of Jus- 1439 tice, Firearm Examiner Training. Accessed via http://www.ojp.usdoj.gov/nij/train- 1440 ing/firearms-training/index.htm 2009 (on 29/04/2009).

55] R.S. Bolton-King, A.R.W. Jackson, Persor Forensic Disciplines: A UK Study, CS Eye April. Accessed via http://www.cseye.com/ 1443 content/2014/april/technical\%20notes/firearms\%20comp 2014 (on 24/06/2015). 1444

[56] AFTE, AFTE Certification Program: AFTE Certification Policies and Procedures, 1445 Accessed via http://afte.org/AssociationInfo/a_certification.htm\#letters 2014 (on 1446 24/06/2015).

[57] AFTE, AFTE Certification Program: AFTE Certified Members List Accessed via http:// 1448 www.afte.org/AssociationInfo/a_certificationmemberslist.htm 2015 (on 24/06/ 1449 2015).

58] The Chartered Society of Forensic Sciences, Frequently Asked Questions. Accessed 1451 via http://www forensic-science-Society.org uk/FAQ 2014 (on 24/06/2015).

[59] The Chartered Society of Forensic Sciences, Register of Experts. Accessed via http:// 1453 www.forensic-science-society.org.uk/ExpertRegister 2015 (on 24/06/2015). 1454

60] AFTE Committee for the Advancement of the Science of Firearm \& Toolmark 1455 Identification, The response of the Association of Firearm and Tool Mark Examiners 1456 
to the National Academy of Sciences 2008 report assessing the feasibility, accuracy, and technical capability of a national ballistics database August 20, 2008, AFTE J. 40 (3) (2008) 234-244.

[61] AFTE Committee for the Advancement of the Science of Firearm \& Toolmark Identification, The response of the association of firearm and tool mark examiners to the February 2009 National Academy of Science report strengthening forensic science in the United States: a path forward, AFTE J. 41 (3) (2009) 204-208.

[62] E.J.A.T. Mattijssen, Examination of firearms review: 2010 to 2013, 17th Interpol International Forensic Science Managers Symposium, Lyon, France 2013, pp. 6-43.

[63] S.G. Bunch, E.D. Smith, B.N. Giroux, D.P. Murphy, Is a match really a match? A primer on the procedures and validity of firearm and toolmark identification, Forensic Sci. Commun. 11 (3) (2009) (June 17, 2015).

[64] W. Kerkhoff, R.D. Stoel, C.E.H. Berger, et al., Design and results of an exploratory double blind testing program in firearms examination, Sci. Justice (2015) (in press).

[65] T.G. Fadul, G.A. Hernandez, E. Wilson, S. Stoiloff, S. Gulati, An Empirical Study to Improve the Scientific Foundation of Forensic Firearm and Tool Mark Identification Utilizing Consecutively Manufactured Glock EBIS Barrels With the Same EBIS Pattern, 244232, Department of Justice, USA, 2013 (Accessed via <https://www. ncjrs.gov/pdffiles1/nij/grants/244232.pdf $\$$ on 24/06/2015).

[66] R. Nichols, Defending the scientific foundations of the firearms and tool mark identification discipline: responding to recent challenges, J. Forensic Sci. 52 (3) (2007) 586-594.

[67] B. Bachrach, A statistical validation of the individuality of guns using 3D images of bullets, U. S. Department of Justice 2136742006 1-65.

[68] T.J. Weller, A. Zheng, R. Thompson, F. Tulleners, Confocal microscopy analysis of breech face marks on fired cartridge cases from 10 consecutively manufactured pistol slides, J. Forensic Sci. 57 (4) (2012) 912-917.

69] A. Stroman, Empirically determined frequency of error in cartridge case examinations using a declared double-blind format, AFTE J. 46 (2) (2014) 157-175.

[70] J.E. Davis, An Introduction to Tool Marks, Firearms and the Striagraph, Thomas, Springfield, Illinois, USA, 1958.
[71] T.B. Brinck, Comparing the performance of IBIS and BulletTRAX-3D technology using 1488 bullets fired through 10 consecutively rifled barrels, J. Forensic Sci. 53 (3) (2008) 1489 $677-682$.

[72] J.F. Song, T.V. Vorburger, T. Renegar, et al., Correlation of topography measurements 1491 of NIST SRM 2460 standard bullets by four techniques, Meas. Sci. Technol. 17 (2006) 1492 500-503.

[73] J. Song, E. Whitenton, D. Kelly, R. Clary, L. Ma, S. Ballou, SRM 2460/2461 standard 1494 bullets and casings project J. Res. Natl. Inst Stand Technol 109 (6) (2004) 533-542. 1495

[74] J. Song, T.V. Vorburger, S. Ballou, et al., The national ballistics imaging comparison 1496 (NBIC) project, Forensic Sci. Int. 216 (1-3) (2012) 168-182.

75] R.S. Bolton-King, J.P.O. Evans, C.L. Smith, J.D. Painter, D.F. Allsop, W.M. Cranton, What 1498 are the prospects of 3D profiling systems applied to firearms and toolmark identifi- 1499 cation? AFTE J. 42 (1) (2010) 23-33.

1500

[76] W. King, W. Wells, C. Katz, E. Maguire, J. Frank, Opening the Black Box of NIBIN: A 1501 Descriptive Process and Outcome Evaluation of the Use of NIBIN and its Effects on 1502 Criminal Investigations, Final Report, 243875, 2013.

[77] J. De Ceuster, R. Hermsen, M. Mastaglio, R. Nennstiel, A discussion on the usefulness 1504 of a shared European ballistic image database, Sci. Justice 52 (4) (2012) 237-242. 1505

[78] S. Bunch, G. Wevers, Application of likelihood ratios for firearm and toolmark anal- 1506 ysis, Sci. Justice 53 (2) (2013) 223-229. 1507

[79] G. Wevers, Are class characteristics really inconclusive? 46th Annual AFTE Training 1508 Seminar, Dallas, Texas, 24-29 May, 2015.

[80] ENFSI, ENFSI Guideline for Evaluative Reporting in Forensic Science: Strengthening 1510 the Evaluation of Forensic Results Across Europe (STEOFRAE), ENFSI, Warsaw, 1511 Poland, 2015.

[81] G. Langenburg, C. Champod, T. Genessay, Informing the judgments of fingerprint an- 1513 alysts using quality metric and statistical assessment tools, Forensic Sci. Int. 2191514 (2012) 183-198.

[82] A. Zheng, J. Soons, A.R.M. Thompson, M. Tong, NIST Reference Ballistic Tool Mark Da- 1516 tabase for Research and Development of Identification Systems and Confidence 1517 Limits, 2015 374-375. 